\title{
Ovarian Serous Carcinoma: A Retrospective Study of Clinicopathological Findings and Postchemotherapy Changes
}

\begin{abstract}
Background: Ovarian carcinoma represents $30 \%$ of all cancers of the female genital tract, of which high-grade serous carcinomas (HGSCs) are predominant, accounting for $70 \%$. Aims and Objectives: To study the clinicopathological findings and to analyze the postchemotherapy changes in tumors treated with neoadjuvant chemotherapy (NACT). Materials and Methods: All cases diagnosed as ovarian serous carcinoma between 2015 and 2017 at our institute were retrospectively reviewed. Clinical and gross findings were collected, microscopic findings were reviewed, and tumor grade was reassessed as per the World Health Organization 2014 criteria. Chemotherapy response score (CRS) was assessed in cases which received prior chemotherapy. Results: Among malignant ovarian tumors, serous carcinoma was the most common, accounting to 38 cases $(44.7 \%)$. Of these, six were low-grade serous carcinoma and 32 were HGSC. Among HGSC, six $(18.75 \%)$ cases showed serous tubal intraepithelial carcinoma. Among 18 (47.4\%) cases with prior NACT, CRS-1 was seen in six cases, CRS-2 in seven cases, and CRS-3 in five cases. Cancer antigen (CA)-125 levels were markedly raised in all cases. In six cases postchemotherapy, CA-125 levels were below normal with a CRS-2-3. Omental deposits were seen in $15(39.47 \%)$ cases and showed lesser response to prior NACT compared to tumor in the ovary. Conclusion: HGSC is the most common ovarian serous carcinoma. There is correlation between the biochemical and morphological response to chemotherapy in our study. Pathologists should be well aware of postchemotherapy morphological changes in ovarian serous carcinoma.
\end{abstract}

Keywords: Ovarian serous carcinoma, postchemotherapy changes, serous tubal intraepithelial carcinoma

\section{Introduction}

Ovarian carcinoma represents $30 \%$ of all cancers of the female genital tract, of which high-grade serous carcinomas (HGSCs) are predominant accounting for $70 \%{ }^{\left[{ }^{[1]}\right.}$ Size of residual disease after surgery and International Federation of Gynecology and Obstetrics (FIGO) stage at diagnosis are the two main prognostic indicators. ${ }^{[2]}$ Most patients present at an advanced stage with a poor survival rate. The treatment of such ovarian cancers is usually maximal surgical debulking followed by chemotherapy. However, preoperative neoadjuvant chemotherapy (NACT) followed by intervention debulking has been the recently followed trend in the management of ovarian cancer. ${ }^{[3]}$ The present study emphasizes the clinicopathological findings as well as postchemotherapy changes in ovarian serous carcinomas.

\footnotetext{
This is an open access journal, and articles are distributed under the terms of the Creative Commons Attribution-NonCommercial-ShareAlike 4.0 License, which allows others to remix, tweak, and build upon the work non-commercially, as long as appropriate credit is given and the new creations are licensed under the identical terms.
}

For reprints contact: WKHLRPMedknow_reprints@wolterskluwer.com

\section{Materials and Methods}

The present study is a 2-year retrospective study which includes cases from July 2015 to June 2017. The study was approved by the ethics committee of our institute. All cases diagnosed as ovarian serous carcinoma were retrospectively reviewed. The study group comprised 38 cases of serous ovarian carcinoma, of which 18 cases with advanced-stage ovarian cancer were treated with preoperative chemotherapy. Clinical and gross findings were collected, microscopic findings were reviewed, and tumor grade and stage were assessed as per the 2014 World Health Organization Classification and the FIGO Surgical Staging System, respectively. ${ }^{[2]}$

Clinicopathological data collected included age at diagnosis, chief complaints, any significant past history or family history of malignancy, cytology of the peritoneal fluid, any lymph node metastasis, FIGO stage,

\footnotetext{
How to cite this article: Vangala N, Uppin SG, Uppin MS, Paul TR, Sadashivudu G. Ovarian serous carcinoma: A retrospective study of clinicopathological findings and postchemotherapy changes. Indian J Med Paediatr Oncol 2020;41:663-9.
}

\section{Navatha Vangala, Shantveer G Uppin, Megha S Uppin, Tara Roshni Paul, G Sadashivudu}

Department of Medical Oncology, Nizam's Institute of Medical Sciences, Hyderabad, Telangana, India

Submitted: 16-Sep-2019 Revised: 28-Mar-2020 Accepted: 09-Jun-2020 Published: 29-Oct-2020

\author{
Address for correspondence: \\ Dr. Shantveer G Uppin, \\ Department of Pathology, \\ Nizam's Institute of Medical \\ Sciences, Hyderabad, \\ Telangana, India. \\ E-mail:drsguppin@yahoo.co.in
}

Access this article online

Website: www.ijmpo.org

DOI: 10.4103/ijmpo.ijmpo_199_19 Quick Response Code:

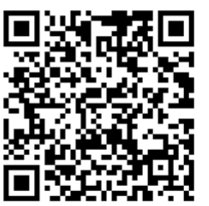


and preoperative chemotherapy regimen and the methods of surgical treatment. Serum cancer antigen (CA)-125 values measured immediately before and after chemotherapy had been noted. The chemotherapy regimen varied between individual patients but usually included three cycles of carboplatin and paclitaxel before debulking surgery followed by another set of three cycles. In 18 cases, diagnosis of adenocarcinoma was confirmed before chemotherapy by cytological examination of the peritoneal fluid. Rest of the cases were diagnosed on tissue biopsies before chemotherapy. Based on the chemotherapy response score (CRS) system criteria proposed by the International Collaboration on Cancer Reporting, CRS was assessed in cases which received NACT before debulking surgery. ${ }^{[3]}$

\section{Results}

A total of 156 ovarian tumors were encountered during the study period. Of these, $85(54.5 \%)$ were malignant neoplasms. Serous carcinoma was the most common of all malignant ovarian tumors, accounting to 38 cases (44.7\%). Of these, six were low-grade serous carcinomas (LGSC) and 32 were HGSCs.

The age at presentation ranged between 24 and 70 years for LGSC and 40 and 71 years for HGSC. Among HGSC cases, two cases had past history of esophageal squamous cell carcinoma (SCC) and one case had a history of SCC in the lung. Bilateral involvement was seen in $16(42 \%)$ cases.

Surface involvement with rupture of ovarian capsule was seen in seven cases. In 15 (39.47\%) cases, omental deposits were seen.

All six cases of LGSC were not treated with preoperative NACT. Histopathology of LGSC revealed a variety of architectural patterns, including single cells and irregularly shaped small nests of cells haphazardly infiltrating stroma, extensive papillae formation with variable fibrovascular stroma. The cells showed minimal nuclear atypia, and few showed a single prominent nucleolus. Frequent psammoma bodies and very occasional mitotic figures were noted [Figure 1a-c]. Two cases showed a component of serous borderline tumor/atypical proliferative serous tumor (SBT/APST). Necrosis was not identified. Ki-67 proliferation index was significantly lower in LGSC when compared to HGSC.

Among 32 cases of HGSC, 14 cases were resected without prior NACT. Histopathology revealed branching papillary fronds and solid masses of the cells with slit-like fenestrations and stratifications. Focal areas showed cribriform and glandular patterns. Cells showed marked pleomorphism with prominent nucleoli and frequent mitoses. Large areas of necrosis and variable number of psammoma bodies were identified [Figure 1d-f].

Immunohistochemistry (IHC) with p53 (6/6) showed intense positivity in the lesional cells of HGSC. Other markers that were significantly expressed in HGSC were cytokeratin 7 (4/4), CA-125 (1/1), Wilms tumor 1 (3/4), and epithelial membrane antigen (1/1). Ki-67 proliferation index was significantly higher $(67 \%)$ in HGSC when compared to LGSC [Figure 2].

As depicted in Table 1, two cases of LGSC and 13 cases of HGSC were in FIGO stage III at the time of presentation. Among HGSC cases in stage III, one case involved the appendix, four cases involved the colon with mesenteric deposits, and one case showed metastatic deposits in the retroperitoneal lymph node.

Preoperative NACT was given in 18 cases of HGSC. Serous tubal intraepithelial carcinoma (STIC) was identified in six $(18.75 \%)$ cases of HGSC with distinct morphological features such as epithelial stratification, moderate to marked nuclear pleomorphism, prominent nucleoli, increased nuclear/cytoplasmic ratio, loss of polarity, and frequent mitotic figures. All cases were ipsilateral. Abnormal p53 expression with high $\mathrm{Ki}-67$ proliferation index $(60 \%)$ was seen in STIC lesions [Figure 3]. The incidence of STIC in cases not treated with prior NACT was $29 \%$ (four cases) compared to $11 \%$ (two cases) in treated cases.

Of the 18 cases who received prior NACT, nearly all cases showed more than $50 \%$ reduction in CA-125 levels, of which 33\% (6 cases) showed below normal CA-125 levels postchemotherapy with CRS-2 and CRS-3. Table 2 shows the serum CA-125 values immediately before and after chemotherapy, the percent drop in CA-125 concentrations, and the CRS suggesting a response to chemotherapy.

CRS was assessed in all the 18 cases who received NACT. Viable tumor with no or minimal response (CRS-1) was seen in six cases, appreciable tumor response amid viable tumor that is readily identifiable (CRS-2) was seen in seven cases, and complete or near-complete response with no residual tumor or minimal irregularly scattered tumor foci (CRS-3) was seen in five cases. In the current study, extensive fibrosis, necrosis with widespread infiltration by chronic inflammatory cells in the tumor, sheets of foamy macrophages, psammoma bodies, hemosiderin-laden macrophages, and marked anisonucleosis with the presence of many bizarre tumor cell nuclei were significantly seen in cases with CRS-2 and CRS-3 when compared to CRS-1 [Figure 4].

\begin{tabular}{lcc}
\hline \multicolumn{2}{c}{$\begin{array}{c}\text { Table 1: International Federation of Gynecology and } \\
\text { Obstetrics stage of low-grade and high-grade serous } \\
\text { carcinomas }\end{array}$} \\
\hline FIGO stage & LGSC, $\boldsymbol{n}(\%)$ & HGSC, $\boldsymbol{n}(\%)$ \\
\hline I & $3(50)$ & $15(46.9)$ \\
II & $1(16.7)$ & $4(12.5)$ \\
III & $2(33.3)$ & $13(40.6)$ \\
Total & $6(16)$ & $32(84)$ \\
\hline
\end{tabular}

FIGO - International Federation of Gynecology and Obstetrics; LGSC - Low-grade serous carcinoma; HGSC - High-grade serous carcinoma 


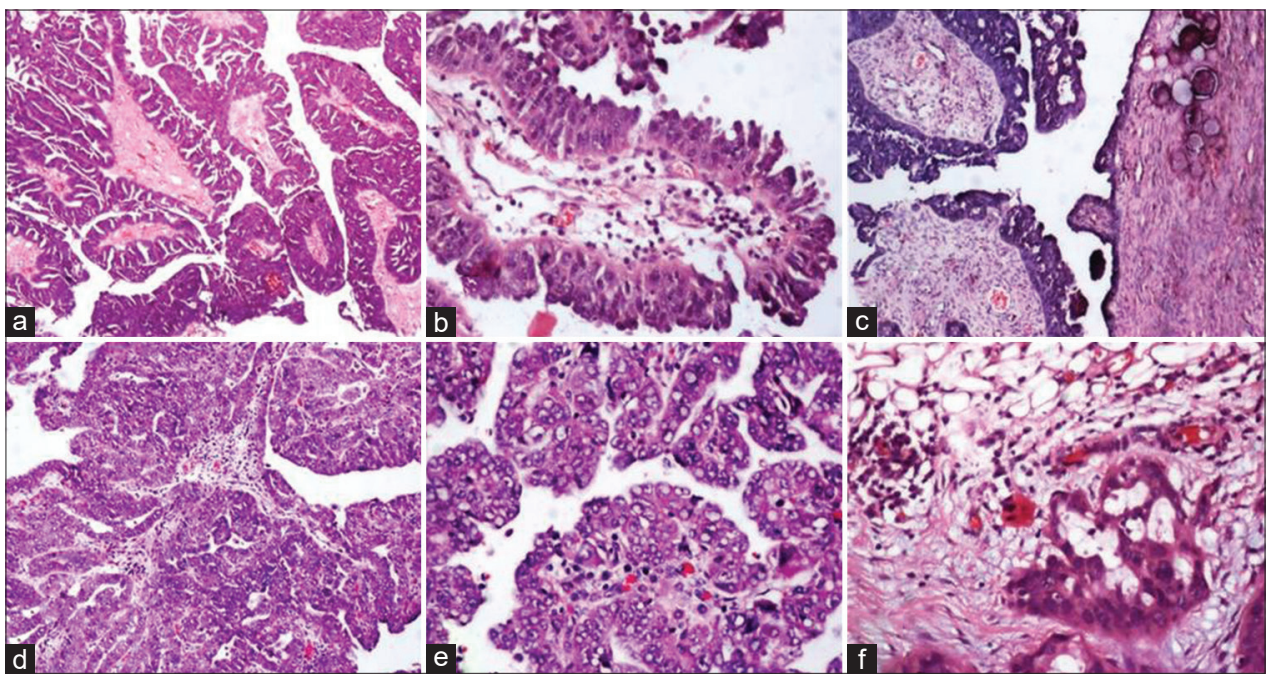

Figure 1: (a-c) Low-grade serous carcinomas; (d-f) high-grade serous carcinomas; (c) surface involvement; (f) omental metastasis (H and E, $\times 100)$

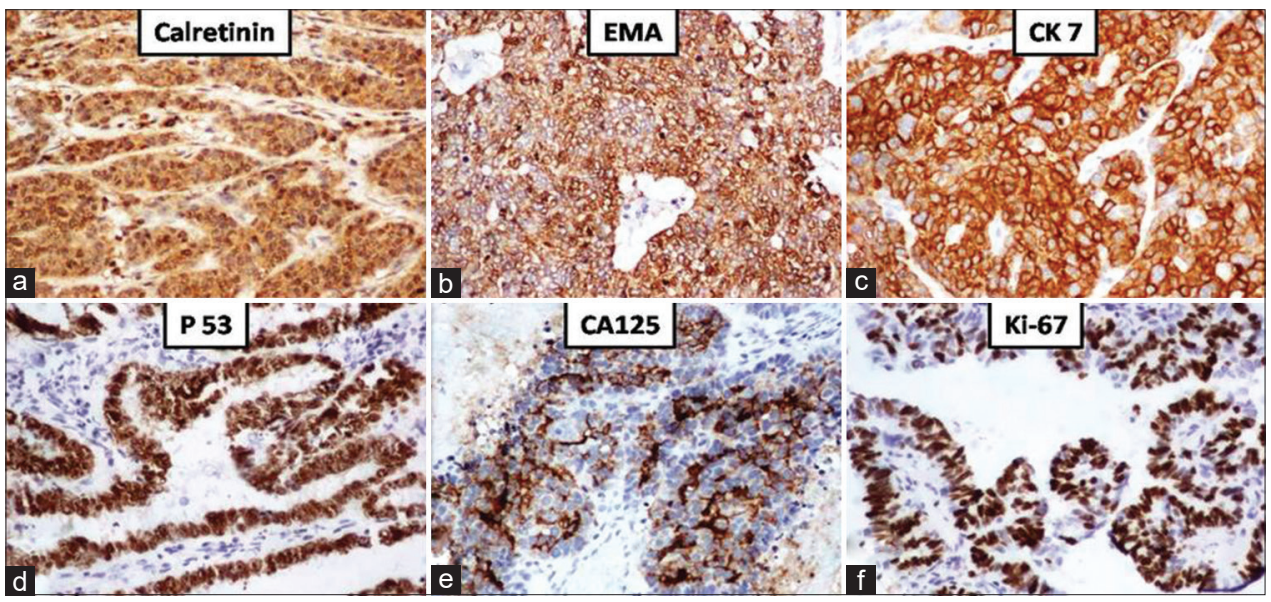

Figure 2: High-grade serous carcinomas: Positive staining by (a) calretinin; (b) epithelial membrane antigen; (c) cytokeratin 7; (d) p53; (e) cancer antigen-125; (f) Ki67 - 67\% (IHC, ×100)

\section{Discussion}

Ovarian cancer is one of the leading causes of morbidity and mortality in females, with a 5-year survival rate of $20 \%-40 \%$ in western nations. ${ }^{[1]}$ Of these, $15 \%-20 \%$ are known to have germline mutations of BRCA1 or BRCA2. The lifetime risk of developing ovarian cancer in women with germline mutations of BRCA is $16 \%-59 \%$ and in women without germline mutations is only $1.4 \% \cdot{ }^{[4,5]}$ Serous carcinoma is the most common ovarian neoplasm which is now considered to represent two separate diseases: LGSC and HGSC. This is based on the recent classification of ovarian cancer into two broad categories, designated type I and II based on molecular genetics and morphologic characteristics. ${ }^{[6]}$

HGSC is the most common subtype of epithelial ovarian cancers and the most aggressive malignancy presenting at advanced stages (stage III or IV) at diagnosis. LGSC accounts for only about $5 \%$ of all serous carcinomas and usually present one decade earlier than HGSC. ${ }^{[7,8]}$
In our study, HGSC accounted for 84\% (32 cases) and the youngest age of presentation for LGSC was 24 years whereas it was 40 years for HGSC.

Factors associated with shorter lifetime number of menstrual cycles such as later age at menarche, earlier age at menopause and oral contraceptive use, ${ }^{[9]}$ greater numbers of births, tubal ligation, ${ }^{[10]}$ especially among BRCA $1 / 2$ mutation carriers, have a protective effect, whereas infertility is associated with a significant risk of ovarian carcinoma. ${ }^{[11-13]}$

Ovarian masses at the initial stages are usually asymptomatic and detected incidentally. Presenting symptoms are relatively nonspecific which could delay the diagnosis, and these include abdominal distension, bloating, pain, constipation, urinary frequency, nausea, and anorexia. ${ }^{[14,15]}$ Cough and dyspnea are the common symptoms in cases with malignant pleural effusion. Abdominal distension with pain, urinary frequency, and nausea were the predominant presenting complaints in our study. 
On imaging, ovarian masses are usually large, complex, cystic pelvic masses with areas of septal thickening or may have solid nodular areas with increased vascularity. In the present study, most of the neoplasms had a complex solid cystic appearance on radiology, with two cases showing adherence and infiltration to rectum and sigmoid colon, respectively. Ascites and omental/peritoneal nodules are

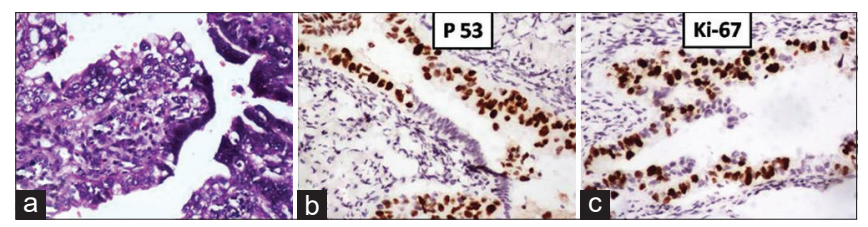

Figure 3: Serous tubal intraepithelial carcinoma: (a) (H and E, ×400); (b) p53 expression (IHC, ×100); (c) Ki67-60\% (IHC, ×100) identified in $15(39.47 \%)$ cases, indicating advanced stage disease with elevated serum biomarkers such as CA-125..$^{[16]}$

LGSC exemplifies the classically held view of a stepwise progression, i.e., adenomacarcinoma sequence, and in our study, two cases showed a component of SBT/APST. ${ }^{[6]}$ As per the recent concepts, majority of HGSC originates from the fimbriated end of the fallopian tube secretory epithelial cells and a small part from cortical inclusion cyst. ${ }^{[17,18]}$ STIC is a high-grade preneoplastic noninvasive lesion, which was first reported by Piek et al., ${ }^{[19]}$ in the fallopian tubes of women with a BRCA1 or BRCA2 mutation, who underwent risk-reducing salpingo-oophorectomies. ${ }^{[20-23]}$ Review of literature shows that in asymptomatic BRCA mutation carriers, the incidence of STIC is $0.6 \%-10 \% .^{[5,24-27]}$ The

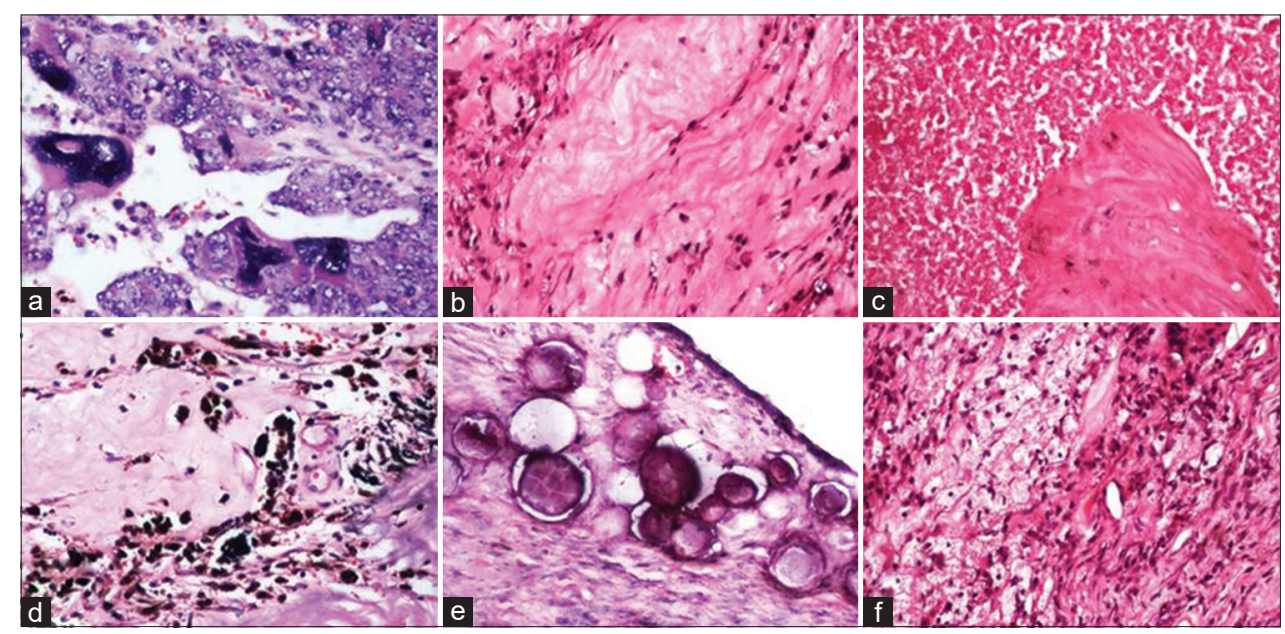

Figure 4: Chemotherapy-induced changes: (a) Bizarre tumor nuclei; (b) extensive fibrosis; (c) tumor necrosis; (d) hemosiderin-laden macrophages; (e) psammoma bodies; (f) foamy macrophages ( $\mathrm{H}$ and $\mathrm{E}, \times 100$ )

\begin{tabular}{|c|c|c|c|c|}
\hline Age (years) & $\begin{array}{c}\text { Serum CA-125 }(\mathrm{U} / \mathrm{ml}) \text { prior } \\
\text { chemotherapy }\end{array}$ & $\begin{array}{c}\text { Serum CA-125 }(\mathrm{U} / \mathrm{ml}) \text { after } \\
\text { chemotherapy }\end{array}$ & $\begin{array}{c}\text { Percentage decrease in serum CA-125 } \\
\text { values }\end{array}$ & CRS score \\
\hline 59 & 3200 & 85 & 97 & 3 \\
\hline 45 & 237 & 12 & 95 & 3 \\
\hline 40 & 107 & 25 & 76 & 2 \\
\hline 58 & 482 & 18 & 96 & 3 \\
\hline 52 & 600 & 124 & 79 & 1 \\
\hline 70 & 4701 & 71 & 98 & 2 \\
\hline 48 & 821 & 92 & 89 & 1 \\
\hline 45 & 88 & 15 & 83 & 2 \\
\hline 45 & 674 & 230 & 66 & 1 \\
\hline 65 & 524 & 192 & 63 & 1 \\
\hline 47 & 653 & 45 & 93 & 2 \\
\hline 55 & 245 & 115 & 54 & 1 \\
\hline 42 & 487 & 76 & 83 & 1 \\
\hline 65 & 354 & 47 & 87 & 3 \\
\hline 38 & 950 & 28 & 97 & 2 \\
\hline 70 & 198 & 13 & 93 & 3 \\
\hline 60 & 1827 & 83 & 94 & 2 \\
\hline 60 & 2147 & 96 & 95 & 2 \\
\hline
\end{tabular}

CA-125 - Cancer antigen-125; CRS - Chemotherapy response score 
frequency of STIC lesions increases with age and is lower with oral contraceptive use. STIC is the earliest known manifestation of most pelvic serous cancers, with an incidence of $11 \%-68 \%$ in HGSC. ${ }^{[28-33]}$ With thorough examination of fallopian tubes by Sectioning and Extensively Examining the Fimbriated End of the Fallopian tube protocol, the incidence of STIC was $35 \%$ in Koc et al.'s study. ${ }^{[34]}$ In our study, STIC was seen in $6(18.75 \%)$ cases of HGSC, of which cases not treated with prior NACT showed significantly higher incidence (four cases) compared to treated cases (two cases). The reduced incidence of STIC in treated cases could be due to its response followed by regression to NACT.

The treatment of advanced-stage ovarian cancer is usually maximal surgical debulking followed by chemotherapy. However, recently, there has been a trend toward preoperative chemotherapy followed by intervention debulking. In our study, $18(47.4 \%)$ cases received prior NACT. As per Hynninen et al., visualization of residual disease following NACT is difficult and less reliable. Therefore, prognostic effect of complete resection is less influential after NACT than after primary surgery. ${ }^{[35]}$

CA-125 levels pre- and post-NACT were assessed in our study. CA-125 levels together with cross-sectional imaging are routinely used to assess response to NACT to determine the suitability for interval debulking surgery (IDS). ${ }^{[36]}$ IDS is not suitable for those who show no CA-125 response to NACT and usually have a poor prognosis. ${ }^{[37]}$ A study by Pelissier et al. highlights the role of CA-125 as a useful marker in prediction of disease progression post-NACT. ${ }^{[38]}$

Table 2 depicts that, in our study, nearly all cases showed more than 50\% reduction in CA-125 levels, of which $33 \%$ showed below normal levels postchemotherapy with a CRS-2 and CRS-3. A study by Rustin et al. demonstrated that CA-125 response does not discriminate between the different grades of pathologic response. ${ }^{[39]}$ There was a correlation between the biochemical response to chemotherapy and the morphological response in our study, emphasizing its role as a useful marker for detecting disease progression. In a study by McCluggage et al., there was no obvious correlation between CA-125 levels postchemotherapy and the morphological response. ${ }^{[40]}$

CRS score is assessed in the omentum as the assessment of adnexal disease after chemotherapy was difficult and less reproducible to score and showed no significant correlation with the outcome. Omentum is proved to be the more prognostically relevant disease site for chemotherapy response assessment as it responds least to chemotherapy. ${ }^{[41]}$ CRS system is easy to apply, reproducible, and prognostically relevant. The grading system recommends the selection of a single block of involved omental tissue that shows the least response to chemotherapy for CRS scoring, and the amount of viable tumor should be assessed on a single hematoxylin and eosin-stained section. ${ }^{[3]}$ Patients with CRS-1 or -2 have a high probability of platinum-refractory disease compared to those with CRS-3. Addition of novel agents such as bevacizumab and/or combinations of agents can improve outcomes in such patients with long-term disease control. ${ }^{[42,43]}$ Whether CRS can be applied to different treatment regimens is not yet determined, but it stands as a potential step toward individualized treatment modification in patients with HGSC postchemotherapy. The present study highlights the importance of CRS system and its incorporation into routine reporting for its prognostic significance. Morphological features of carcinoma ovary following NACT differ markedly from those of the native neoplasm. Boehm et al. developed a new simple three-tier CRS scoring system for assessing histopathological response to NACT. ${ }^{[3]}$ It has been used in ovarian cancer reporting by the International Collaboration on Cancer Reporting as reported by McCluggage et al. ${ }^{[40]}$ Assessment of CRS has to be done in the omentum as it responds least to chemotherapy. ${ }^{[41]}$ Extensive fibrosis, necrosis with widespread infiltration by chronic inflammatory cells in the tumor, sheets of foamy macrophages, psammoma bodies, hemosiderin-laden macrophages, and marked anisonucleosis with the presence of many bizarre tumor cell nuclei were significantly seen in cases with CRS-2 and CRS-3 when compared to CRS-1.

\section{Conclusion}

HGSC is the most common and the most aggressive ovarian carcinoma that usually presents at an advanced stage. There is correlation between the biochemical and morphological response to chemotherapy. Pathologist should be aware of chemotherapy-induced morphological features, and proper sampling of the entire tumor area followed by assessment of chemotherapy response is highly recommended due to its prognostic significance.

\section{Financial support and sponsorship}

Nil.

\section{Conflicts of interest}

There are no conflicts of interest.

\section{References}

1. Ferlay J, Shin HR, Bray F, Forman D, Mathers C, Parkin DM. Estimates of worldwide burden of cancer in 2008: GLOBOCAN 2008. Int J Cancer 2010;127:2893-917.

2. Prat J. FIGO Committee on Gynecologic Oncology. Staging classification for cancer of the ovary, fallopian tube, and peritoneum. Int J Gynecol Obstet 2014;124:1-5.

3. Boehm S, Said I, Faruqi A, Gilks CB, Singh N. Development of a response scoring system to quantify the effect of neoadjuvant chemotherapy in ovarian cancer response scoring study. Modern Pathol 2014;27:276A.

4. Nishida N, Murakami F, Higaki K. Detection of serous precursor lesions in resected fallopian tubes from patients with benign diseases and a relatively low risk for ovarian cancer. Pathol Int 2016;66:337-42. 
5. Zeppernick F, Meinhold-Heerlein I, Shih IE. Precursors of ovarian cancer in the fallopian tube: Serous tubal intraepithelial carcinoma-An update. J Obstet Gynaecol Re 2015;41:6-11.

6. Kobayashi H, Iwai K, Niiro E, Morioka S, Yamada Y, Ogawa K, et al. The conceptual advances of carcinogenic sequence model in high-grade serous ovarian cancer. Biomed Rep 2017;7:209-13.

7. Köbel M, Kalloger SE, Huntsman DG, Santos JL, Swenerton KD, Seidman JD, et al. Differences in tumor type in low-stage versus high-stage ovarian carcinomas. Int J Gynecol Pathol 2010;29:203-11.

8. Vang R, Shih IeM, Kurman RJ. Ovarian low-grade and high-grade serous carcinoma: Pathogenesis, clinicopathologic and molecular biologic features, and diagnostic problems. Adv Anat Pathol 2009;16:267-82.

9. Collaborative Group on Epidemiological Studies of Ovarian Cancer, Beral V, Doll R, Hermon C, Peto R, Reeves G. Ovarian cancer and oral contraceptives: Collaborative reanalysis of data from 45 epidemiological studies including 23,257 women with ovarian cancer and 87,303 controls. Lancet 2008;371:303-14.

10. Cibula D, Widschwendter M, Májek O, Dusek L. Tubal ligation and the risk of ovarian cancer: Review and meta-analysis. Hum Reprod Update 2011;17:55-67.

11. Hunn J, Rodriguez GC. Ovarian cancer: Etiology, risk factors, and epidemiology. Clin Obstet Gynecol 2012;55:3-23.

12. Pelucchi C, Galeone C, Talamini R, Bosetti C, Montella M, Negri E, et al. Lifetime ovulatory cycles and ovarian cancer risk in two Italian case-control studies. Am J Obstet Gynecol 2007;196:83-7.

13. Sueblinvong T, Carney ME. Current understanding of risk factors for ovarian cancer. Curr Treat Option On 2009;10:67-81.

14. Goff BA, Mandel LS, Melancon CH, Muntz HG. Frequency of symptoms of ovarian cancer in women presenting to primary care clinics. JAMA 2004;291:2705-12.

15. Gilbert L, Basso O, Sampalis J, Karp I, Martins C, Feng J, et al. Assessment of symptomatic women for early diagnosis of ovarian cancer: Results from the prospective DOvE pilot project. Lancet Oncol 2012;13:285-91.

16. Zivanovic O, Sima CS, Iasonos A, Bell-McGuinn KM, Sabbatini PJ, Leitao MM, et al. Exploratory analysis of serum CA-125 response to surgery and the risk of relapse in patients with FIGO stage IIIC ovarian cancer. Gynecol Oncol 2009;115:209-14.

17. George SH, Garcia R, Slomovitz BM. Ovarian cancer: The fallopian tube as the site of origin and opportunities for prevention. Front Oncol 2016;6:108.

18. Wang J, Wu H, Zhang Y, Zhang Y, Li X, Zhao Q, et al. High-grade serous ovarian and fallopian tube carcinomas with similar clinicopathological characteristics might originate from serous tubal intraepithelial carcinoma in Chinese women. Int $J$ Clin Exp Pathol 2017;10:8222-32.

19. Piek JM, van Diest PJ, Zweemer RP, Jansen JW, Poort-Keesom RJ, Menko FH, et al. Dysplastic changes in prophylactically removed fallopian tubes of women predisposed to developing ovarian cancer. J Pathol 2001;195:451-6.

20. Powell CB, Chen LM, McLennan J, Crawford B, Zaloudek C, Rabban JT, et al. Risk-reducing salpingo-oophorectomy (RRSO) in BRCA mutation carriers: Experience with a consecutive series of 111 patients using a standardized surgical-pathological protocol. Int J Gynecol Cancer 2011;21:846-51.

21. Reitsma W, De Bock GH, Oosterwijk JC, Bart J, Hollema H, Mourits MJ. Support of the 'fallopian tube hypothesis' in a prospective series of risk-reducing salpingo-oophorectomy specimens. Eur J Cancer 2013;49:132-41.
22. George SH, Greenaway J, Milea A, Clary V, Shaw S, Sharma M, et al. Identification of abrogated pathways in fallopian tube epithelium from BRCA1 mutation carriers. J Pathol 2011;225:106-17.

23. Tone AA, Virtanen C, Shaw P, Brown TJ. Prolonged postovulatory pro-inflammatory signaling in the fallopian tube epithelium may be mediated through a BRCA1/DAB2 axis. Clin Cancer Res 2012;18:4334-44.

24. Malmberg K, Klynning C, Flöter-Rådestad A, Carlson JW. Serous tubal intraepithelial carcinoma, chronic fallopian tube injury, and serous carcinoma development. Virchows Arch 2016;468:707-13.

25. Tang S, Onuma K, Deb P, Wang E, Lytwyn A, Sur M, Daya D. Frequency of serous tubal intraepithelial carcinoma in various gynecologic malignancies: A study of 300 consecutive cases. Int J Gynecol Pathol 2012;31:103-10.

26. Mittal N, Srinivasan R, Gupta N, Rajwanshi A, Nijhawan R, Gautam U, et al. Secretory cell outgrowths, p53 signatures, and serous tubal intraepithelial carcinoma in the fallopian tubes of patients with sporadic pelvic serous carcinoma. Indian J Pathol Microbiol 2016;59:481-8.

27. Chen F, Gaitskell K, Garcia MJ, Albukhari A, Tsaltas J, Ahmed AA. Serous tubal intraepithelial carcinomas associated with high-grade serous ovarian carcinomas: A systematic review. BJOG 2017;124:872-8.

28. Schneider S, Heikaus S, Harter P, Heitz F, Grimm C, Ataseven B, et al. Serous tubal intraepithelial carcinoma associated with extraovarian metastases. Int J Gynecol Cancer 2017;27:444-51.

29. Corzo C, Iniesta MD, Patrono MG, Lu KH, Ramirez PT. Role of fallopian tubes in the development of ovarian cancer. J Minim Invas Gyn 2017;24:230-4.

30. Perets R, Wyant GA, Muto KW, Bijron JG, Poole BB, Chin KT, et al. Transformation of the fallopian tube secretory epithelium leads to high- grade serous ovarian cancer in BRCA; Tp53; PTEN models. Cancer Cell 2013;24:751-65.

31. Kaur KK, Allahbadia G, Singh M. An update on high grade serous ovarian carcinoma-A comprehensive review. Acta Sci Cancer Biol 2019;3:37-49.

32. Lisio MA, Fu L, Goyeneche A, Gao ZH, Telleria C. High-grade serous ovarian cancer: Basic sciences, clinical and therapeutic standpoints. Int J Mol Sci 2019;20::952.

33. Vang R, Shih IeM, Kurman RJ. Fallopian tube precursors of ovarian low- and high-grade serous neoplasms. Histopathology 2013;62:44-58.

34. Koc N, Ayas S, Arinkan SA. Comparison of the classical method and SEE-FIM protocol in detecting microscopic lesions in fallopian tubes with gynecological lesions. J Pathol Transl Med 2018;52:21-7.

35. Hynninen J, Lavonius M, Oksa S, Grénman S, Carpén O, Auranen A. Is perioperative visual estimation of intra-abdominal tumor spread reliable in ovarian cancer surgery after neoadjuvant chemotherapy? Gynecol Oncol 2013;128:229-32.

36. Ledermann JA, Raja FA, Fotopoulou C, Gonzalez-Martin A, Colombo N, Sessa C, et al. Newly diagnosed and relapsed epithelial ovarian carcinoma: ESMO Clinical Practice Guidelines for diagnosis, treatment and follow-up. Ann Oncol 2013;24 Suppl 6:vi24-32.

37. Saha A, Varughese M, Gallagher CJ, Orphanos G, Wilson P, Oram $\mathrm{D}$, et al. Primary chemotherapy for inoperable ovarian, fallopian tube, or primary peritoneal cancer with or without delayed debulking surgery. Int J Gynecol Cancer 2012;22:566-72.

38. Pelissier A, Bonneau C, Chéreau E, de La Motte Rouge T, Fourchotte V, Daraï E, et al. CA125 kinetic parameters predict 
optimal cytoreduction in patients with advanced epithelial ovarian cancer treated with neoadjuvant chemotherapy. Gynecol Oncol 2014;135:542-6.

39. Rustin GJ, Vergote I, Eisenhauer E, Pujade-Lauraine E, Quinn M, Thigpen T, et al. Definitions for response and progression in ovarian cancer clinical trials incorporating RECIST 1.1 and CA 125 agreed by the Gynecological Cancer Intergroup (GCIG). Int J Gynecol Cancer 2011;21:419-23.

40. McCluggage WG, Judge MJ, Clarke BA, Davidson B, Gilks CB, Hollema $\mathrm{H}$, et al. Data set for reporting of ovary, fallopian tube and primary peritoneal carcinoma: Recommendations from the International Collaboration on Cancer Reporting (ICCR). Mod
Pathol 2015;28:1101-22.

41. Leinster DA, Kulbe H, Everitt G, Thompson R, Perretti M, Gavins FNE, et al. The peritoneal tumor microenvironment of high-grade serous ovarian cancer. J Pathol 2012;227:136-45.

42. Gourley C, McCavigan A, Perren T, Paul J, Michie CO, Churchman $\mathrm{M}$, et al. Molecular subgroup of high-grade serous ovarian cancer (HGSOC) as a predictor of outcome following bevacizumab. J Clin Oncol 2014;5:32.

43. Riester M, Wei W, Waldron L, Culhane AC, Trippa L, Oliva E, et al. Risk prediction for late-stage ovarian cancer by metaanalysis of 1525 patient samples. J Natl Cancer I 2014;106. pii: Dju048. 